\title{
N $91-16884$
}

\section{HI and Mass Distribution of GR8}

\author{
Sylvie Beaulieu, Space Telescope Science Institute \\ and \\ Claude Carignan, Université de Montréal
}

\begin{abstract}
The dwarf irregular galaxy GR8, which is at the extreme faint end of the luminosity and mass functions, is studied using optical photometry and $21 \mathrm{~cm}$ HI line observations. It is shown that rotation is only important to the gravitational support of the system in the inner parts $(\mathrm{r}<250 \mathrm{pc})$. GR8 is one of the very few non-elliptical systems known (with M81dwA) where the random motions provide essentially all the support in the outer parts $(r \geq 500 \mathrm{pc})$. The Gaussian nature of the HI distribution and the isothermal distribution of the HI velocity dispersion implies $M \propto R^{3}$ in the outer regions of GR8 (ie the stellar disk and the HI lie in the approximately uniform density core of the dark halo).
\end{abstract}

\section{Introduction}

A few years ago, a program was undertaken to extend the mass and luminosity range of galaxies with well studied and understood mass distribution. The technique consists essentially in getting the kinematics to large galactocentric distances using high sensitivity HI observations and to complement this with optical surface photometry studies. The HI kinematics allows us to derive the total mass distribution (out to the last measured velocity point) in 
the system while the surface photometry tells us about the mass contribution of the luminous stellar disk. The data is usually analyzed in terms of a twocomponent model: a maximum luminous stellar disk and a minimum dark halo represented by an isothermal sphere potential or some other similar forms.

We have already reported the results on three late-type spirals in the Sculptor Group with $M_{B} \approx-18$, and on the Magellanic-type spiral NGC 3109 at $M_{B} \approx-17$ (Carignan and Freeman 1985). More recently, the luminosity range was extended toward still fainter systems with the study of the dwarf "regular" galaxy UGC 2259 at $\mathrm{M}_{\mathrm{B}} \approx-16.5$ (Carignan et al. 1988), and the dwarf irregular galaxy DDO 154 at $\mathrm{M}_{\mathrm{B}} \approx-14$ (Carignan and Beaulieu 1989; Carignan and Freeman 1988). It is with the goal of extending this kind of study to the extreme faint end of the luminosity and mass functions of normal galaxies, that we undertook the present study of GR. $\left(M_{B} \approx-11\right)$

\section{Optical observations}

$\rightarrow$ Well defined luminosity profiles were derived in $B, V$, and $R$, which show very regular exponential decline for $r \geq 30^{\prime \prime}$. An exponential fit to the $B$ profile yields an extrapolated central surface brightness $\mathrm{B}(0)_{\mathrm{c}}=22.6 \pm 0.2$ mag $\operatorname{arcsec}^{-2}$ and a scale length of $76 \pm 7 \mathrm{pc}(\Delta=1.1 \mathrm{Mpc})$.

$\rightarrow$ The colors are not as blue as previously suggested $((B-V) \simeq 0.06$; Hodge 1967) but are more typical of the expected colors for Im galaxies, with $(B-V)=0.38$.

\section{HI line observations}

$\rightarrow$ The velocity width $\Delta V_{20}=37 \mathrm{~km} \mathrm{~s}^{-1}$ indicates that rotation is present in 
this system: the velocity field shows random motions with a fairly constant velocity dispersion $\sigma \approx 9.8 \pm 2.2 \mathrm{~km} \mathrm{~s}^{-1}$.

$\rightarrow$ By integrating the global profile, a total HI mass of $2.0 \pm 0.2 \times 10^{6} \mathrm{M}_{\odot}$ is derived, for a $M_{H} / L_{B}=0.8$.

$\rightarrow$ The HI distribution is clumpy, with the clumps located just outside the optical structure. Its radial distribution is well approximated by a Gaussian having a scale length of $268 \mathrm{pc}$ along the kinematical major axis.

$\rightarrow$ The most striking result from the kinematical analysis is that the gas is rotating with its rotation axis roughly parallel and not perpendicular to the common major axis of the optical and HI distributions.

\section{Mass distribution}

$\rightarrow$ For the stellar disk, the colors suggest a $M_{*} / L_{B} \simeq 0.5$ which, when combined with the observed total luminosity, yield a stellar mass of $\mathrm{M}_{*} \simeq 1.2$ $\times 10^{6} M_{\odot}$. Combining the mass in stars with our derived HI mass gives a total luminous mass of $\mathrm{M}_{\mathrm{lum}}=3.2 \times 10^{6} \mathrm{M}_{\odot}$.

$\rightarrow$ From Table I, it can be seen that rotation is only important in the inner parts $(R<250 \mathrm{pc})$ and that, in the outer parts $(R \approx 500 \mathrm{pc})$, random motions are really what provide the gravitational support to the system. Contrary to a common misconception, GR 8 and M81dwA (Sargent, Sancisi, and Lo 1983) are the only two non-elliptical systems where this is observed.

$\rightarrow$ The total dynamical mass within $R=500 \mathrm{pc}$ is $3.9 \times 10^{7} \mathrm{M}_{\odot}$, which strongly indicates that the luminous component of GR8 is immersed in the usual clark halo. The integrated $M_{\text {global }} / L_{B}$ ratio within $0.5 \mathrm{kpc}$ is 16 . 


\section{Conclusion}

The mean density of the dark halo of GR8 $\left(\left\langle\rho_{\text {dark }}\right\rangle \simeq 0.07 \mathrm{M}_{\odot} \mathrm{pc}^{-3}\right)$ appears to be unusually high compared to the densities of halos seen in spirals. However, it is comparable to what is seen in dwarf spheroidals (Kormendy 1987). The limited extent of the III distribution means that little more can be said about its other parameters, such as its core radius and total mass. However, the Gaussian nature of the III distribution and the isothermal distribution of the HI velocity dispersion implics that $M \propto R^{3}$ in the outer regions of GR8 (Carignan, Beaulieu, and Freeman 1990).

This suggests that the luminous matter (stars \& HI) lies within the approximately uniform density region of the core of the dark halo. This feature is not peculiar to extreme dwarls such as GR8. For example, an analysis of the rotation curve of the dwarf spiral UGC 2259 (Carignan et al. 1988) shows again that most of the luminous matter lies within the nearly constant density core region of its dark halo.

Our results reinforce the view that cven the faintest dwarfs have dark halos (Carignan and Beaulieu 1989; Skillman el al. 1987; Sargent, Sancisi, and Lo 1983). They also support earlier suggestions (Kormendy 1987; Carignan and Freeman 1988) that the halos of the faintest dwarfs are densest. 


\section{REFERENCES}

Carignan, C., and Freeman, K.C. (1985). Astrophys.J. 299, 59.

Carignan, C., and Freeman, K.C. (1988). Astrophys.J.Lett. 332, L33.

Carignan, C., Sancisi, R., and Albada., T.S. van (1988). Astron.J. 95, 37.

Carignan, C., and Beaulieu, S. (1989). Astrophys.J. 347, in press.

Carignan, C., Beaulieu, S., and Freeman, K.C. (1990). Astron.J., in press.

Hodge, P.W. (1967). Astrophys.J. 148, 719.

Kormendy, J. (1987). In Dark Matter in the Universe, ed. J. Kormendy and G.R. Knapp (Dordrecht, Reidel), p. 139.

Sargent, W.L.W., Sancisi, R., and Lo, K.-Y. (1983). Astrophys.J. 265, 711.

Skillman, E.D., Bothun, G.D., Murray, M.A., and Warmels, R.H. (1987). Astron. Astrophys. 185, 61 .

TABLE I. MASS ESTIMATES FOR GR8

\begin{tabular}{ccc}
\hline \hline $\mathrm{R}(\mathrm{pc})$ & $\mathrm{M}(\mathrm{R})\left(\mathrm{M}_{\odot}\right)$ & $f_{\text {rot }}^{\dagger}$ \\
$(1)$ & $(2)$ & $(3)$ \\
\hline & & \\
125 & $2.2 \times 10^{6}$ & 0.73 \\
250 & $6.9 \times 10^{6}$ & 0.32 \\
500 & $3.9 \times 10^{7}$ & 0.03 \\
\hline
\end{tabular}

${ }^{\dagger} f_{\text {rot }}$ is the fractional contribution of rotation to the support of GR8. 